Bull. Korean Math. Soc. 51 (2014), No. 4, pp. 1041-1054

http://dx.doi.org/10.4134/BKMS.2014.51.4.1041

\title{
GENERALIZED FIBONACCI AND LUCAS NUMBERS OF THE FORM $w x^{2}$ AND $w x^{2} \mp 1$
}

\author{
REFiK KESKiN
}

\begin{abstract}
Let $P \geq 3$ be an integer and let $\left(U_{n}\right)$ and $\left(V_{n}\right)$ denote generalized Fibonacci and Lucas sequences defined by $U_{0}=0, U_{1}=1$; $V_{0}=2, V_{1}=P$, and $U_{n+1}=P U_{n}-U_{n-1}, V_{n+1}=P V_{n}-V_{n-1}$ for $n \geq 1$. In this study, when $P$ is odd, we solve the equations $V_{n}=k x^{2}$ and $V_{n}=2 k x^{2}$ with $k \mid P$ and $k>1$. Then, when $k \mid P$ and $k>1$, we solve some other equations such as $U_{n}=k x^{2}, U_{n}=2 k x^{2}, U_{n}=3 k x^{2}, V_{n}=$ $k x^{2} \mp 1, V_{n}=2 k x^{2} \mp 1$, and $U_{n}=k x^{2} \mp 1$. Moreover, when $P$ is odd, we solve the equations $V_{n}=w x^{2}+1$ and $V_{n}=w x^{2}-1$ for $w=2,3,6$. After that, we solve some Diophantine equations.
\end{abstract}

\section{Introduction}

Let $P$ and $Q$ be nonzero integers. Generalized Fibonacci sequence $\left(U_{n}\right)$ and Lucas sequence $\left(V_{n}\right)$ are defined by $U_{0}(P, Q)=0, U_{1}(P, Q)=1 ; V_{0}(P, Q)=$ $2, V_{1}(P, Q)=P$, and $U_{n+1}(P, Q)=P U_{n}(P, Q)+Q U_{n-1}(P, Q), V_{n+1}(P, Q)=$ $P V_{n}(P, Q)+Q V_{n-1}(P, Q)$ for $n \geq 1 . U_{n}(P, Q)$ and $V_{n}(P, Q)$ are called $n$-th generalized Fibonacci number and $n$-th generalized Lucas number, respectively. Generalized Fibonacci and Lucas numbers for negative subscripts are defined as $U_{-n}(P, Q)=-(-Q)^{-n} U_{n}(P, Q)$ and $V_{-n}(P, Q)=(-Q)^{-n} V_{n}(P, Q)$, respectively.

Now assume that $P^{2}+4 Q \neq 0$. Then it is well known that

$$
U_{n}=U_{n}(P, Q)=\frac{\alpha^{n}-\beta^{n}}{\alpha-\beta} \text { and } V_{n}=V_{n}(P, Q)=\alpha^{n}+\beta^{n},
$$

where $\alpha=\frac{P+\sqrt{P^{2}+4 Q}}{2}$ and $\beta=\frac{P-\sqrt{P^{2}+4 Q}}{2}$, which are the roots of the characteristic equation $x^{2}-P x-Q=0$.

The above formulas are known as Binet's formulas. Since

$$
U_{n}(-P, Q)=(-1)^{n-1} U_{n}(P, Q) \text { and } V_{n}(-P, Q)=(-1)^{n} V_{n}(P, Q),
$$

Received August 11, 2013; Revised November 25, 2013.

2010 Mathematics Subject Classification. 11B37, 11B39, 11B50, 11B99, $11 \mathrm{D} 41$.

Key words and phrases. generalized Fibonacci numbers, generalized Lucas numbers, congruences, Diophantine equation. 
it will be assumed that $P \geq 1$. Moreover, we will assume that $P^{2}+4 Q>0$. For $P=Q=1$, we have classical Fibonacci and Lucas sequences $\left(F_{n}\right)$ and $\left(L_{n}\right)$. For $P=2$ and $Q=1$, we have Pell and Pell-Lucas sequences $\left(P_{n}\right)$ and $\left(Q_{n}\right)$. For more information about generalized Fibonacci and Lucas sequences one can consult $[4,10,11,12]$.

Generalized Fibonacci and Lucas numbers of the form $k x^{2}$ have been investigated since 1962. When $P$ is odd and $Q=\mp 1$, by using elementary argument, many authors solved the equations $U_{n}=k x^{2}$ or $V_{n}=k x^{2}$ for specific integer values of $k$. The reader can consult [17] for a brief discussion of this subject. When $P$ and $Q$ are relatively prime odd integers, in [13], the authors solved $U_{n}=x^{2}, U_{n}=2 x^{2}, V_{n}=x^{2}, V_{n}=2 x^{2}$. Moreover, under the same assumption, in [15], the same authors solved $U_{n}=3 x^{2}$ and they solved $V_{n}=k x^{2}$ under some assumptions on $k$.

In [2], when $P$ is odd, Cohn solved the equations $V_{n}=P x^{2}$ and $V_{n}=$ $2 P x^{2}$ with $Q=\mp 1$. When $P$ is odd, in [17], the authors solved the equation $V_{n}(P, 1)=k x^{2}$ for $k \mid P$ with $k>1$. In this study, when $P$ is odd, we will solve the equations $V_{n}(P,-1)=k x^{2}$ and $V_{n}(P,-1)=2 k x^{2}$ for $k \mid P$ with $k>1$. Then, when $k \mid P$ with $k>1$, we will solve some other equations such as $U_{n}(P,-1)=k x^{2}, U_{n}(P,-1)=2 k x^{2}, U_{n}(P,-1)=3 k x^{2}, V_{n}(P,-1)=k x^{2} \mp 1$, $V_{n}(P,-1)=2 k x^{2} \mp 1$, and $U_{n}(P,-1)=k x^{2} \mp 1$. When $P$ is odd, we will solve the equations $V_{n}(P,-1)=w x^{2}+1$ and $V_{n}(P,-1)=w x^{2}-1$ for $w=2,3,6$. Thus we solve some Diophantine equations.

We will use the Jacobi symbol throughout this study. Our method is elementary and used by Cohn, Ribenboim and McDaniel in [2] and [15], respectively.

\section{Preliminaries}

From now on, sometimes, instead of $U_{n}(P,-1)$ and $V_{n}(P,-1)$, we will use $U_{n}$ and $V_{n}$, respectively. Moreover, we will assume that $P \geq 3$. The following lemmas can be proved by induction.

Lemma 2.1. If $n$ is a positive integer, then $V_{2 n} \equiv \mp 2\left(\bmod P^{2}\right)$ and $V_{2 n+1} \equiv$ $(2 n+1) P(-1)^{n}\left(\bmod P^{2}\right)$.

Lemma 2.2. If $n$ is a positive integer, then $U_{2 n} \equiv n(-1)^{n+1} P\left(\bmod P^{2}\right)$ and $U_{2 n+1} \equiv(-1)^{n}\left(\bmod P^{2}\right)$.

The following lemma is given in [13] and [15].

Lemma 2.3. $\left(\frac{U_{3}}{V_{2^{r}}}\right)=1$ for $r \geq 1$.

The following lemma is a consequence of a theorem given in [6].

Lemma 2.4. All positive integer solutions of the equation $3 x^{2}-2 y^{2}=1$ are given by $(x, y)=\left(U_{n}(10,-1)-U_{n-1}(10,-1), U_{n}(10,-1)+U_{n-1}(10,-1)\right)$ with $n \geq 1$.

The following theorems are well known (see $[3,5,8,9])$. 
Theorem 2.5. All positive integer solutions of the equation $x^{2}-\left(P^{2}-4\right) y^{2}=4$ are given by $(x, y)=\left(V_{n}(P,-1), U_{n}(P,-1)\right)$ with $n \geq 1$.

Theorem 2.6. All positive integer solutions of the equation $x^{2}-P x y+y^{2}=1$ are given by $(x, y)=\left(U_{n}(P,-1), U_{n-1}(P,-1)\right)$ with $n \geq 1$.

The proofs of the following two theorems are given in [16].

Theorem 2.7. Let $n \in \mathbb{N} \cup\{0\}, m, r \in \mathbb{Z}$ and $m$ be a nonzero integer. Then

$$
U_{2 m n+r} \equiv U_{r} \quad\left(\bmod U_{m}\right)
$$

and

$$
V_{2 m n+r} \equiv V_{r} \quad\left(\bmod U_{m}\right) .
$$

Theorem 2.8. Let $n \in \mathbb{N} \cup\{0\}$ and $m, r \in \mathbb{Z}$. Then

$$
U_{2 m n+r} \equiv(-1)^{n} U_{r} \quad\left(\bmod V_{m}\right)
$$

and

$$
V_{2 m n+r} \equiv(-1)^{n} V_{r} \quad\left(\bmod V_{m}\right)
$$

When $P$ is odd, since $8 \mid U_{3}$, using (3) we get

$$
V_{6 q+r} \equiv V_{r} \quad(\bmod 8) \text {. }
$$

Thus

$$
4 \nmid V_{n} .
$$

Moreover, an induction method shows that

$$
V_{2^{r}} \equiv 7 \quad(\bmod 8)
$$

and thus

$$
\left(\frac{2}{V_{2^{r}}}\right)=1
$$

for $r \geq 1$.

When $P$ is odd, it is seen that

$$
\left(\frac{-1}{V_{2^{r}}}\right)=-1
$$

for $r \geq 1$.

Secondly, we give some identities concerning generalized Fibonacci and Lucas numbers:

$$
\begin{gathered}
U_{-n}=-U_{n} \text { and } V_{-n}=V_{n}, \\
U_{2 n+1}-1=U_{n} V_{n+1}, \\
U_{2 n}=U_{n} V_{n}, \\
V_{2 n}=V_{n}^{2}-2,
\end{gathered}
$$




$$
U_{3 n}=U_{n}\left(\left(P^{2}-4\right) U_{n}^{2}+3\right)=U_{n}\left(V_{n}^{2}-1\right)=U_{n}\left(V_{2 n}+1\right),
$$

$$
V_{3 n}=V_{n}\left(V_{n}^{2}-3\right)=V_{n}\left(V_{2 n}-1\right),
$$

$$
\text { If } P \text { is odd, then } 2\left|V_{n} \Leftrightarrow 2\right| U_{n} \Leftrightarrow 3 \mid n \text {, }
$$

$$
V_{n}^{2}-\left(P^{2}-4\right) U_{n}^{2}=4 .
$$

Let $m=2^{a} k, n=2^{b} l, k$ and $l$ odd, $a, b \geq 0$, and $d=(m, n)$. Then

$$
\left(U_{n}, U_{m}\right)=U_{d},
$$

$$
\left(U_{m}, V_{n}\right)=\left\{\begin{array}{c}
V_{d} \quad \text { if } a>b, \\
1 \text { or } 2 \quad \text { if } a \leq b .
\end{array}\right.
$$

If $P$ is odd and $r \geq 2$, then $V_{2^{r}} \equiv-1\left(\bmod \frac{P^{2}-3}{2}\right)$ and thus

$$
\left(\frac{\left(P^{2}-3\right) / 2}{V_{2^{r}}}\right)=\left(\frac{P^{2}-3}{V_{2^{r}}}\right)=1 .
$$

$$
\text { If } r \geq 1 \text {, then } V_{2^{r}} \equiv \mp 2 \quad(\bmod P) \text {. }
$$

$$
\text { If } r \geq 2 \text {, then } V_{2^{r}} \equiv 2 \quad(\bmod P) .
$$

If $3 \nmid P$, then $3 \mid U_{3}$. Thus we get

$$
3\left|U_{n} \Leftrightarrow 3\right| n
$$

by $(2)$ and $V_{2^{r}} \equiv-1(\bmod 3)$ and therefore

$$
\left(\frac{3}{V_{2^{r}}}\right)=1
$$

for $r \geq 1$.

If $3 \mid P$ and $P$ is odd, then $V_{2^{r}} \equiv-1(\bmod 3)$ for $r \geq 2$ and thus

$$
\left(\frac{3}{V_{2^{r}}}\right)=1
$$

for $r \geq 2$. Moreover, we have

$$
\left(\frac{P-1}{V_{2^{r}}}\right)=\left(\frac{P+1}{V_{2^{r}}}\right)=1
$$

for $r \geq 1$.

Identities in between (11)-(16) and (17)-(18) can be found in $[12,15,16]$ and $[7,14,15]$, respectively. The proofs of the others are easy and will be omitted. 


\section{Main theorems}

From now on, we will assume that $n$ is a positive integer and $P$ is odd.

Lemma 3.1. Let $m>1$ be odd. Then $V_{2 m}+1=2 x^{2}$ has no solutions.

Proof. Assume that $V_{2 m}+1=2 x^{2}$ for some integer $x$. Let $2 m=2(4 q \mp 1)=$ $2\left(2^{r} a \mp 1\right)$ with $a$ odd and $r \geq 2$. Thus

$$
2 x^{2}=V_{2 m}+1 \equiv 1-V_{2} \equiv-\left(P^{2}-3\right) \quad\left(\bmod V_{2^{r}}\right)
$$

by (5), which implies that

$$
x^{2} \equiv-\left(\frac{P^{2}-3}{2}\right) \quad\left(\bmod V_{2^{r}}\right) .
$$

This shows that

$$
\left(\frac{\left(P^{2}-3\right) / 2}{V_{2^{r}}}\right)=-1
$$

which is impossible by (19).

Theorem 3.2. If $V_{n}=k x^{2}$ for some $k \mid P$ with $k>1$, then $n=1$.

Proof. Assume that $V_{n}=k x^{2}$ for some $k \mid P$ with $k>1$. Then by Lemma 2.1 , it is seen that $n$ is odd. Let $n=6 q+r$ with $r \in\{1,3,5\}$. Then by (6), $V_{n}=V_{6 q+r} \equiv V_{r}(\bmod 8)$ and therefore $V_{n} \equiv V_{1}, V_{3}, V_{5}(\bmod 8)$. It is seen that $V_{n} \equiv P, 6 P(\bmod 8)$. Then $k x^{2} \equiv P, 6 P(\bmod 8)$. Let $P=k M$. Thus, we get $k M x^{2} \equiv P M, 6 P M(\bmod 8)$, which implies that $P x^{2} \equiv P M, 6 P M(\bmod 8)$. This shows that $x^{2} \equiv M, 6 M(\bmod 8)$ since $P$ is odd. Therefore $M \equiv 1$ $(\bmod 8)$ since $M$ is odd. Now assume that $n>1$. Then $n=4 q \mp 1=2^{r+1} a \mp 1$ with $a$ odd and $r \geq 1$. Since $V_{n}=k x^{2}$, we get

$$
k x^{2}=V_{n} \equiv-V_{\mp 1} \equiv-P \quad\left(\bmod V_{2^{r}}\right)
$$

by (5). This shows that

$$
x^{2} \equiv-M \quad\left(\bmod V_{2^{r}}\right)
$$

which implies that

$$
\left(\frac{-1}{V_{2^{r}}}\right)\left(\frac{M}{V_{2^{r}}}\right)=1
$$

By using the fact that $M \equiv 1(\bmod 8)$, we get

$$
\left(\frac{M}{V_{2^{r}}}\right)=\left(\frac{V_{2^{r}}}{M}\right)=\left(\frac{\mp 2}{M}\right)=1
$$

by $(20)$. Since $\left(\frac{-1}{V_{2^{r}}}\right)=-1$ by $(9)$, we get $\left(\frac{-1}{V_{2^{r}}}\right)\left(\frac{M}{V_{2^{r}}}\right)=-1$, which contradicts (27). Therefore $n=1$.

We can give the following corollary by using Theorems 2.5 and 3.2 . 
Corollary 3.3. The only positive integer solution of the equation $P^{2} x^{4}-\left(P^{2}-\right.$ 4) $y^{2}=4$ is $(x, y)=(1,1)$.

Theorem 3.4. If $V_{n}=2 k x^{2}$ for some $k \mid P$ with $k>1$, then $n=3$.

Proof. Assume that $V_{n}=2 k x^{2}$ for some $k \mid P$ with $k>1$. Then $3 \mid n$ and $n$ is odd by (15) and Lemma 2.1. Let $n=3 m$ with $m$ odd. Then $2 k x^{2}=$ $V_{n}=V_{3 m}=V_{m}\left(V_{m}^{2}-3\right)$ by $(14)$ and thus $\left(V_{m} / k\right)\left(V_{m}^{2}-3\right)=2 x^{2}$. Since $\left(V_{m}, V_{m}^{2}-3\right)=1$ or 3 , we get $V_{m}^{2}-3=w a^{2}$ for some $w \in\{1,2,3,6\}$. Since $V_{m}^{2}-3=V_{2 m}-1$, it is seen that $V_{2 m}-1=w a^{2}$. Assume that $m>1$. Then $m=4 q \mp 1=2^{r} a \mp 1$ with $a$ odd and $r \geq 2$. Thus,

$$
w a^{2}=V_{2 m}-1 \equiv-1-V_{\mp 2} \equiv-1-\left(P^{2}-2\right) \equiv-\left(P^{2}-1\right) \quad\left(\bmod V_{2^{r}}\right) .
$$

This shows that

$$
\left(\frac{w}{V_{2^{r}}}\right)=\left(\frac{-1}{V_{2^{r}}}\right)\left(\frac{P^{2}-1}{V_{2^{r}}}\right) .
$$

By using (8), (23), and (24), it can be seen that $\left(\frac{w}{V_{2^{r}}}\right)=1$ for $w=2,3,6$. Moreover, $\left(\frac{-1}{V_{2^{r}}}\right)=-1$ and $\left(\frac{P^{2}-1}{V_{2^{r}}}\right)=1$ by (9) and Lemma 2.3, respectively. Thus, we get

$$
1=\left(\frac{w}{V_{2^{r}}}\right)=\left(\frac{-1}{V_{2^{r}}}\right)\left(\frac{P^{2}-1}{V_{2^{r}}}\right)=-1,
$$

which is impossible. Therefore $m=1$ and thus, $n=3$.

We can give the following corollary easily.

Corollary 3.5. The equation $4 P^{2} x^{4}-\left(P^{2}-4\right) y^{2}=4$ has no integer solutions.

Proof. Assume that $4 P^{2} x^{4}-\left(P^{2}-4\right) y^{2}=4$ for some positive integer $x$ and $y$. Then by Theorems 2.5 and 3.4 , we get $V_{3}=2 P x^{2}$. This shows that $2 P x^{2}=$ $P\left(P^{2}-3\right)$, which implies that $P^{2}-2 x^{2}=3$. But this is impossible.

Now we give some known theorems from [13], which will be useful for solving the equations $U_{n}=k x^{2}, U_{n}=2 k x^{2}$, and $U_{n}=3 k x^{2}$, where $k \mid P$ with $k>1$. We use a theorem from [1] while solving $V_{n}=2 x^{2}$.

Theorem 3.6. If $V_{n}=x^{2}$ for some integer $x$, then $n=1$. If $V_{n}=2 x^{2}$ for some integer $x$, then $n=3, P=3,27$.

Theorem 3.7. If $U_{n}=x^{2}$ for some integer $x$, then $n=1$ or $n=2, P=\square$ or $n=6, P=3$. If $U_{n}=2 x^{2}$ for some integer $x$, then $n=3$.

Theorem 3.8. Let $P \geq 3$ be odd. If $U_{n}=k x^{2}$ for some $k \mid P$ with $k>1$, then $n=2$ or $n=6$ and $3 \mid P$.

Proof. Assume that $U_{n}=k x^{2}$ for some $k \mid P$ with $k>1$. Then by Lemma 2.2, $n$ is even and therefore $n=2 m$ for some positive integer $m$. Thus, $k x^{2}=U_{n}=$ 
$U_{2 m}=U_{m} V_{m}$. Assume that $m$ is even. Then we get $\left(U_{m} / k\right) V_{m}=x^{2}$. In this case, since $\left(U_{m}, V_{m}\right)=1$ or 2 , by (18) either

$$
U_{m}=k u^{2} \text { and } V_{m}=v^{2},
$$

or

$$
U_{m}=2 k u^{2} \text { and } V_{m}=2 v^{2}
$$

for some integers $u$ and $v$. Since $m$ is even, the identities (28) and (29) are impossible by Theorem 3.6. Now assume that $m$ is odd. In this case, either

$$
U_{m}=u^{2} \text { and } V_{m}=k v^{2},
$$

or

$$
U_{m}=2 u^{2} \text { and } V_{m}=2 k v^{2}
$$

for some integers $u$ and $v$. If (30) is satisfied, then $m=1$ by Theorem 3.7 and therefore $n=2$. Assume that (31) is satisfied. Then $m=3$ by Theorems 3.4 and 3.7. Thus $n=6$. In which case, it can be seen that if $U_{6}=U_{3} V_{3}=k x^{2}$ for some $k \mid P$ with $k>1$, then $3 \mid P$.

Corollary 3.9. The equation $P^{2} x^{4}-P^{2} x^{2} y+y^{2}=1$ has only the positive solution $(x, y)=(1,1)$.

Proof. Assume that $P^{2} x^{4}-P^{2} x^{2} y+y^{2}=1$ for some positive integers $x$ and $y$. Then $P x^{2}=U_{2}$ or $P x^{2}=U_{6}$ by Theorems 2.6 and 3.8. It can be seen that $P x^{2}=U_{6}$ is impossible. Therefore $P x^{2}=U_{2}$ and thus $y=U_{1}=1$. This shows that $(x, y)=(1,1)$.

Theorem 3.10. If $k \mid P$ with $k>1$, then $U_{n}=2 k x^{2}$ has no solutions.

Proof. Assume that $U_{n}=2 k x^{2}$. Then $n=6 m$ for some positive integer $m$ by Lemma 2.2 and (15). Thus, we get $U_{n}=U_{6 m}=U_{3 m} V_{3 m}=2 k x^{2}$, which implies that $\left(U_{3 m} / k\right)\left(V_{3 m} / 2\right)=x^{2}$ or $\left(V_{3 m} / 2 k\right) U_{3 m}=x^{2}$. Since $\left(U_{3 m}, V_{3 m}\right)=2$ and $4 \nmid V_{3 m}$ by (7), we get either

$$
U_{3 m}=k u^{2} \text { and } V_{3 m}=2 v^{2}
$$

or

$$
V_{3 m}=2 k u^{2} \text { and } U_{3 m}=v^{2}
$$

for some integers $u$ and $v$. But (32) is impossible by Theorems 3.6 and 3.8 . Moreover (33) is impossible by Theorems 3.4 and 3.7.

Corollary 3.11. The equations $4 P^{2} x^{4}-2 P^{2} x^{2} y+y^{2}=1$ and $x^{2}-4 P^{2}\left(P^{2}-\right.$ 4) $y^{4}=4$ have no integer solutions.

Theorem 3.12. Let $k \mid P$ with $k>1$. If $3 \nmid P$, then $U_{n}=3 k x^{2}$ has no solutions. 
Proof. Assume that $U_{n}=3 k x^{2}$. Since $3 \nmid P$, we get $n=3 m$ for some even integer $m$ by (22) and Lemma 2.2. Thus, $3 k x^{2}=U_{3 m}=U_{m}\left(V_{2 m}+1\right)$ by (13), which implies that $\left(U_{m} / k\right)\left(V_{2 m}+1\right)=3 x^{2}$. Since $\left(U_{m} / k, V_{2 m}+1\right)=1$ or 3 by (13), it follows that $V_{2 m}+1=w u^{2}$ for some $w \in\{1,3\}$. Since $m$ is even, $m=2^{r} a$ with $a$ odd and $r \geq 1$. Thus $w u^{2}=V_{2 m}+1 \equiv 1-V_{0}\left(\bmod V_{2^{r}}\right)$ by $(5)$, which implies that $w u^{2} \equiv-1\left(\bmod V_{2^{r}}\right)$. This is impossible since $\left(\frac{-1}{V_{2^{r}}}\right)=-1$ by $(9)$ and $\left(\frac{3}{V_{2^{r}}}\right)=1$ by $(23)$.

Corollary 3.13. The equations $9 P^{2} x^{4}-3 P^{2} x^{2} y+y^{2}=1$ and $x^{2}-9 P^{2}\left(P^{2}-\right.$ 4) $y^{4}=4$ have no integer solutions.

Theorem 3.14. If $k \mid P$ with $k>1$, then the equation $V_{n}=k x^{2}+1$ has no solutions.

Proof. Assume that $V_{n}=k x^{2}+1$ for some integer $x$. Then by Lemma 2.1, $n$ is even. Let $n=2 m$. If $m$ is even, then we get $n=4 u$, which implies that $V_{n} \equiv 2\left(\bmod U_{2}\right)$ by $(3)$. Therefore $k x^{2}+1 \equiv 2(\bmod P)$, which is impossible since $k \mid P$ and $k>1$. Thus, $m$ is odd. Since $V_{2 m}=V_{m}^{2}-2$ by (12), we get $k x^{2}+1=V_{m}^{2}-2$, which implies that $k \mid 3$ by Lemma 2.1 and therefore $k=3$. Since $k=3$, we see that $3 \mid P$. Assume that $m>1$. Then $m=4 q \mp 1=2^{r} a$ $\mp 1$ with $a$ odd and $r \geq 2$. So, $3 x^{2}+1=V_{n}=V_{2 m} \equiv-V_{\mp 2}\left(\bmod V_{2^{r}}\right)$ by $(5)$. This shows that $3 x^{2} \equiv-\left(P^{2}-1\right)\left(\bmod V_{2^{r}}\right)$. That is, $3 x^{2} \equiv-U_{3}\left(\bmod V_{2^{r}}\right)$. Thus, by using Lemma 2.3, (9), and (24), we get

$$
1=\left(\frac{3}{V_{2^{r}}}\right)=\left(\frac{-U_{3}}{V_{2^{r}}}\right)=\left(\frac{-1}{V_{2^{r}}}\right)\left(\frac{U_{3}}{V_{2^{r}}}\right)=-1,
$$

which is impossible. Therefore $m=1$ and thus, $n=2$. So, $3 x^{2}+1=V_{2}=$ $P^{2}-2$, which is impossible.

Corollary 3.15. The equation $\left(P x^{2}+1\right)^{2}-\left(P^{2}-4\right) y^{2}=4$ has no solutions.

Theorem 3.16. If $k \mid P$ with $k>1$, then the equation $U_{n}=k x^{2}+1$ has only the solution $n=1$.

Proof. Assume that $U_{n}=k x^{2}+1$ for some integer $x$. Then by Lemma $2.2, n$ is odd. Let $n=2 m+1$. Assume that $m>0$. By using (10), we get $k x^{2}=$ $U_{2 m+1}-1=U_{m} V_{m+1}$. Assume that $m$ is odd. Then by $(18),\left(U_{m}, V_{m+1}\right)=1$. Thus $U_{m}=k_{1} u^{2}$ and $V_{m+1}=k_{2} v^{2}$ with $k_{1} k_{2}=k$. This is impossible by Theorems 3.2 and 3.8 , since $k_{1}>1$ or $k_{2}>1$. Now assume that $m$ is even. Then $\left(U_{m}, V_{m+1}\right)=P$ by (18). Thus it follows that $U_{m}=k_{1} P u^{2}$ and $V_{m+1}=k_{2} P v^{2}$ with $k_{1} k_{2}=k$. This is impossible by Theorem 3.2. Therefore $m=0$ and thus $n=1$.

Corollary 3.17. The equations $\left(P x^{2}+1\right)^{2}-P\left(P x^{2}+1\right) y+y^{2}=1$ and $x^{2}-\left(P^{2}-4\right)\left(P y^{2}+1\right)^{2}=4$ have no positive integer solutions. 
By using the fact that $U_{2 m+1}+1=U_{m+1} V_{m}$, we can give the following theorem easily.

Theorem 3.18. If $k \mid P$ with $k>1$, then the equation $U_{n}=k x^{2}-1$ has only the solution $n=3$.

If $P=3$, then $V_{4}=47=3 x^{2}-1$ has solution $x=4$. Now we give the following theorem.

Theorem 3.19. If $k \mid P$ with $k>1$, then the equation $V_{n}=k x^{2}-1$ has only the solution $(n, P, k, x)=(4,3,3,4)$.

Proof. Assume that $V_{n}=k x^{2}-1$ for some integer $x$. Then by Lemma 2.1, $n$ is even. Let $n=2 m$. Then $k x^{2}-1=V_{n}=V_{2 m}=V_{m}^{2}-2$ by (12). Assume that $m$ is odd. Then $P \mid V_{m}$ by Lemma 2.1 , which implies that $k \mid 1$, a contradiction. Therefore $m$ is even and thus $n=4 u$, which shows that $k x^{2}-1=V_{n} \equiv 2$ $\left(\bmod U_{2}\right)$ by $(3)$. This shows that $k=3$ and therefore $3 \mid P$. In this case we have $n=4 u=2\left(2^{r} a\right)$ for some odd integer $a$ with $r \geq 1$ and thus, $3 x^{2}-1 \equiv-2$ $\left(\bmod V_{2^{r}}\right)$ by $(5)$. This shows that $3 x^{2} \equiv-1\left(\bmod V_{2^{r}}\right)$. Therefore

$$
\left(\frac{3}{V_{2^{r}}}\right)=\left(\frac{-1}{V_{2^{r}}}\right)=-1,
$$

which is impossible if $r \geq 2$ by (24). Therefore $r=1$. Then $n=4 u$ with $u$ odd. Thus, $3 x^{2}-1 \equiv V_{4 u}=V_{2 u}^{2}-2$, which implies that $V_{2 u}^{2}-1=3 x^{2}$. That is, $\left(V_{2 u}-1\right)\left(V_{2 u}+1\right)=3 x^{2}$. Let $\left(V_{2 u}-1, V_{2 u}+1\right)=1$. Then we have either

$$
V_{2 u}-1=a^{2} \text { and } V_{2 u}+1=3 b^{2}
$$

$$
V_{2 u}-1=3 a^{2} \text { and } V_{2 u}+1=b^{2}
$$

for some integers $a$ and $b$. It can be seen that (35) and (36) are impossible. Let $\left(V_{2 u}-1, V_{2 u}+1\right)=2$. Then we have

$$
V_{2 u}-1=2 a^{2} \text { and } V_{2 u}+1=6 b^{2}
$$

or

$$
V_{2 u}-1=6 a^{2} \text { and } V_{2 u}+1=2 b^{2}
$$

for some integers $a$ and $b$. It can be shown that (37) is impossible. If $u>1$, then (38) is impossible by Lemma 3.1. Therefore $u=1$ and so $n=4$. Thus $V_{4}=3 x^{2}-1$ and therefore $V_{2}^{2}-2=3 x^{2}-1$. This implies that $\left(P^{2}-2\right)^{2}-3 x^{2}=1$. Since all positive integer solutions of the equation $u^{2}-3 v^{2}=1$ are given by $(u, v)=\left(V_{n}(4,-1) / 2, U_{n}(4,-1)\right)$ with $n \geq 1$, we get

$$
P^{2}-2=\frac{V_{n}(4,-1)}{2}
$$

for some natural number $n$. Thus, $V_{n}(4,-1)=2 P^{2}-4$, which shows that $n$ is even. Let $n=2 m$. Then $2 P^{2}-4=V_{2 m}(4,-1)=V_{m}^{2}(4,-1)-2$. For the time being, we use $V_{n}$ instead of $V_{n}(4,-1)$. If $m$ is even, then $m=2 t$ and 
so $\left(V_{t}^{2}-2\right)^{2}-2=2 P^{2}-4$. This shows that $V_{t}^{4}-4 V_{t}^{2}+6=2 P^{2}$, which is impossible since $V_{t}$ is even. Therefore $m$ is odd. Assume that $m>1$. Then $n=2 m=2(4 q \mp 1)=2\left(2^{r} a \mp 1\right)$ with $a$ odd and $r \geq 2$. Thus,

$$
2 P^{2}-4=V_{n} \equiv-V_{2} \equiv-14 \quad\left(\bmod V_{2^{r}}\right)
$$

which implies that

$$
P^{2} \equiv-5 \quad\left(\bmod V_{2^{r}} / 2\right) .
$$

A simple computation shows that $V_{2^{r}} / 2 \equiv 1(\bmod 4)$ and $V_{2^{r}} / 2 \equiv 2(\bmod 5)$. Thus, we obtain

$$
\left(\frac{-5}{V_{2^{r}} / 2}\right)=\left(\frac{5}{V_{2^{r}} / 2}\right)=\left(\frac{V_{2^{r}} / 2}{5}\right)=\left(\frac{2}{5}\right)=-1,
$$

which is impossible by (39). Therefore $m=1$ and thus $n=2$. This implies that $2 P^{2}-4=V_{n}(4,-1)=V_{2}(4,-1)=14$. Hence we get $P=3$. Since $\left(P^{2}-2\right)^{2}-3 x^{2}=1$, it follows that $x=4$. This completes the proof.

We can give the following corollary easily.

Corollary 3.20. The only positive integer solutions of the equation $\left(P x^{2}-\right.$ $1)^{2}-\left(P^{2}-4\right) y^{2}=4$ is given by $(P, x, y)=(3,4,21)$.

Theorem 3.21. The equation $V_{n}=3 x^{2}-1$ has the solutions

$$
(n, P, x)=(4,3,4),(n, P, x)=\left(1,3 a^{2}-1, a\right)
$$

with a even or $(n, P, x)=\left(2, V_{2 t}(4,-1) / 2, U_{2 t}(4,-1)\right)$ with $t \geq 1$.

Proof. If $3 \mid P$, then by Theorem 3.19, we get $(n, P, k, x)=(4,3,3,4)$. Assume that $3 \nmid P$. Let $n>1$ be odd. Then $n=4 q \mp 1=2^{r+1} b \mp 1$ with $b$ odd and $r \geq 1$. Thus,

$$
3 x^{2}=V_{n}+1 \equiv-V_{1}+1 \equiv-(P-1) \quad\left(\bmod V_{2^{r}}\right)
$$

by (5). By using (23), (9), and (25), it is seen that

$$
1=\left(\frac{3}{V_{2^{r}}}\right)=\left(\frac{-1}{V_{2^{r}}}\right)\left(\frac{P-1}{V_{2^{r}}}\right)=-1,
$$

which is impossible. Therefore $n=1$. And so, $P=3 a^{2}-1$ with $a$ even. Now let $n$ be even. Then $n=2 m$ for some positive integer $m$. Assume that $m>1$ and $m$ is odd. Then $n=2 m=2(4 q \mp 1)=2\left(2^{r} a \mp 1\right)$ with $a$ odd and $r \geq 2$. Thus,

$$
3 x^{2}=1+V_{n} \equiv 1-V_{2} \equiv-\left(P^{2}-3\right) \quad\left(\bmod V_{2^{r}}\right),
$$

by (5). By using (23), (9), and (19), we get

$$
1=\left(\frac{3}{V_{2^{r}}}\right)=\left(\frac{-1}{V_{2^{r}}}\right)\left(\frac{P^{2}-3}{V_{2^{r}}}\right)=-1,
$$

which is a contradiction. Therefore $m=1$ and so $n=2$. Thus $3 x^{2}-1=V_{2}=$ $P^{2}-2$ and this implies that $P^{2}-3 x^{2}=1$. Therefore $P=V_{2 t}(4,-1) / 2$ with 
$t \geq 1$. Assume that $m$ is even. Then $n=4 u=2^{r+1} b$ with $b$ odd and $r \geq 1$. This implies by (5) that

$$
3 x^{2}=1+V_{n} \equiv 1-V_{0} \equiv-1 \quad\left(\bmod V_{2^{r}}\right),
$$

which is impossible since $\left(\frac{-1}{V_{2^{r}}}\right)=-1$ and $\left(\frac{3}{V_{2^{r}}}\right)=1$ by (9) and (23).

Corollary 3.22. The equation $\left(3 x^{2}-1\right)^{2}-\left(P^{2}-4\right) y^{2}=4$ has integer solutions only when $P=3, P=3 a^{2}-1$ with a even or $P=V_{2 t}(4,-1) / 2$ with $t \geq 1$.

Theorem 3.23. Let $k \mid P$ with $k>1$. Then the equation $V_{n}=2 k x^{2}-1$ has no solutions.

Proof. Assume that $V_{n}=2 k x^{2}-1$ for some integer $x$. Then by Lemma 2.1, $n$ is even. Let $n=2 m$. Then $2 k x^{2}-1=V_{n}=V_{2 m}=V_{m}^{2}-2$ by (12). Assume that $m$ is odd. Then $P \mid V_{m}$ by Lemma 2.1, which implies that $k \mid 1$, a contradiction. Therefore $m$ is even and so $n=4 u$, which shows that $2 k x^{2}-1=V_{n} \equiv 2$ $\left(\bmod U_{2}\right)$ by $(3)$. This implies that $k=3$ and therefore $3 \mid P$. In this case, we have $n=4 u=2^{r+1} a$ with $a$ odd and $r \geq 1$. Thus $6 x^{2}-1=V_{n} \equiv-2$ $\left(\bmod V_{2^{r}}\right)$. This shows that $6 x^{2} \equiv-1\left(\bmod V_{2^{r}}\right)$. Therefore

$$
\left(\frac{2}{V_{2^{r}}}\right)\left(\frac{3}{V_{2^{r}}}\right)=\left(\frac{-1}{V_{2^{r}}}\right)=-1,
$$

which is impossible if $r \geq 2$ by (8) and (24). Therefore $r=1$. Then $n=4 u$ with $u$ odd. Thus, $6 x^{2}-1=V_{4 u}=V_{2 u}^{2}-2$, which implies that $V_{2 u}^{2}-1=6 x^{2}$. That is, $\left(V_{2 u}-1\right)\left(V_{2 u}+1\right)=6 x^{2}$. Since $\left(V_{2 u}-1, V_{2 u}+1\right)=1$ or 2 , we have one of the following cases:

$$
\begin{aligned}
& V_{2 u}-1=2 a^{2} \text { and } V_{2 u}+1=3 b^{2}, \\
& V_{2 u}-1=a^{2} \text { and } V_{2 u}+1=6 b^{2}, \\
& V_{2 u}-1=6 a^{2} \text { and } V_{2 u}+1=b^{2}, \\
& V_{2 u}-1=3 a^{2} \text { and } V_{2 u}+1=2 b^{2} .
\end{aligned}
$$

A simple argument shows that (40), (41), and (42) are impossible. Assume that (43) is satisfied. Then $3 a^{2}=V_{2 u}-1=V_{u}^{2}-3$ and so $a^{2}+1=3\left(V_{2 u} / 3\right)^{2}$, which is impossible.

Corollary 3.24. The equation $\left(2 P x^{2}-1\right)^{2}-\left(P^{2}-4\right) y^{2}=4$ has no integer solutions.

Theorem 3.25. The equation $V_{n}=6 x^{2}-1$ has the solutions

$$
(n, P, x)=\left(2, V_{t}(10,-1) / 2, U_{t}(10,-1)\right)
$$

with $t \geq 1$ or $(n, P, x)=\left(1,6 a^{2}-1, a\right)$ with a integer. 
Proof. If $3 \mid P$, then the proof follows from Theorem 3.23. Assume that $3 \nmid P$. Let $n>1$ be odd. Then $n=4 q \mp 1=2^{r+1} b \mp 1$ with $b$ odd and $r \geq 1$. Thus we get

$$
6 x^{2}=V_{n}+1 \equiv-V_{1}+1 \equiv-(P-1) \quad\left(\bmod V_{2^{r}}\right)
$$

by (5). By using (8), (23), (9), and (25), it is seen that

$$
1=\left(\frac{2}{V_{2^{r}}}\right)\left(\frac{3}{V_{2^{r}}}\right)=\left(\frac{-1}{V_{2^{r}}}\right)\left(\frac{P-1}{V_{2^{r}}}\right)=-1,
$$

which is impossible. Therefore $n=1$. And so, $P=6 a^{2}-1$ with $a$ integer. Let $n$ be even, i.e., $n=2 m$. Assume that $m>1$ is odd. Then $m=4 q \mp 1=2^{r} b \mp 1$ with $b$ odd and $r \geq 2$ and thus,

$$
6 x^{2}=V_{n}+1 \equiv-V_{2}+1 \equiv-\left(P^{2}-3\right) \quad\left(\bmod V_{2^{r}}\right)
$$

by (5). Similarly, by using (8), (9), (23), and (19), we get a contradiction. Therefore $m=1$ and so $n=2$. If $m$ is even, we get $n=2^{r+1} b$ with $b$ odd and $r \geq 1$ and thus

$$
6 x^{2}=V_{n}+1 \equiv-V_{0}+1 \equiv-1 \quad\left(\bmod V_{2^{r}}\right),
$$

which is a contradiction by (8), (9), and (23). Therefore $n=2$, which implies that $P^{2}-6 x^{2}=1$. Therefore $P=V_{t}(10,-1) / 2$ and $x=U_{t}(10,-1)$ with $t \geq 1$. Therefore $P=V_{t}(10,-1) / 2$ is a solution.

Corollary 3.26. The equation $\left(6 x^{2}-1\right)^{2}-\left(P^{2}-4\right) y^{2}=4$ has integer solutions only when $P=6 a^{2}-1$ with a integer or $P=V_{m}(10,-1) / 2$ with $m \geq 1$.

Theorem 3.27. Let $k \mid P$ with $k>1$. Then the equation $V_{n}=2 k x^{2}+1$ has the solutions $(n, P, x)=\left(2,3\left(U_{m}(10,-1)-U_{m-1}(10,-1)\right), U_{m}(10,-1)+\right.$ $\left.U_{m-1}(10,-1)\right)$ with $m \geq 1$.

Proof. Assume that $V_{n}=2 k x^{2}+1$. Then $n$ is even by Lemma 2.1. Let $n=2 m$. If $m$ is even, then we get $2 k x^{2}+1=V_{n} \equiv 2\left(\bmod U_{2}\right)$, which is impossible. Therefore $m$ is odd. Since $2 k x^{2}+1=V_{m}^{2}-2$, it follows that $k \mid 3$ and therefore $k=3$. Assume that $m>1$. Then $m=4 q \mp 1=2^{r} a \mp 1$ with $a$ odd and $r \geq 2$. Thus, we get

$$
6 x^{2}=V_{n}-1=V_{2 m}-1 \equiv-V_{2}-1 \equiv-\left(P^{2}-1\right) \quad\left(\bmod V_{2^{r}}\right),
$$

which is impossible by (8), (9), (23), and Lemma 2.3. Then $m=1$ and therefore $n=2$. Thus $6 x^{2}+1=V_{2}=P^{2}-2$. That is, $P^{2}-6 x^{2}=3$. Let $P=3 a$. Then $3 a^{2}-2 x^{2}=1$. By Lemma 2.4, we get

$$
\left.(a, x)=\left(U_{m}(10,-1)-U_{m-1}(10,-1)\right), U_{m}(10,-1)+U_{m-1}(10,-1)\right)
$$

and therefore

$$
(n, P, x)=\left(2,3\left(U_{m}(10,-1)-U_{m-1}(10,-1)\right), U_{m}(10,-1)+U_{m-1}(10,-1)\right)
$$

with $m \geq 1$. 
Corollary 3.28. The equation $\left(2 P x^{2}+1\right)^{2}-\left(P^{2}-4\right) y^{2}=4$ has positive integer solutions only when $P=3\left(U_{m}(10,-1)-U_{m-1}(10,-1)\right)$ with $m \geq 1$.

We can give the following theorems easily.

Theorem 3.29. The equation $V_{n}=3 x^{2}+1$ has no solutions.

Theorem 3.30. The equation $V_{n}=6 x^{2}+1$ has the solutions

$$
(n, P, x)=\left(2,3\left(U_{m}(10,-1)-U_{m-1}(10,-1)\right), U_{m}(10,-1)+U_{m-1}(10,-1)\right)
$$

with $m \geq 1$.

Theorem 3.31. The equation $V_{n}=2 x^{2}-1$ has the solutions

$$
(n, P, x)=\left(2, V_{m}(6,-1) / 2,2 U_{m}(6,-1)\right)
$$

with $m \geq 1$ or $(n, P, x)=\left(1,2 a^{2}-1, a\right)$ with a integer.

Theorem 3.32. The equation $V_{n}=2 x^{2}+1$ has the solution $(n, P, x)=$ $\left(1,2 a^{2}+1, a\right)$ with a integer.

Theorem 3.33. The equation $V_{n}=x^{2}+1$ has the solution $(n, P, x)=\left(1,4 a^{2}+\right.$ 1,2a) with a integer.

Theorem 3.34. The equation $V_{n}=x^{2}-1$ has the solution $(n, P, x)=\left(1,4 a^{2}-\right.$ $1,2 a)$ with a integer.

Corollary 3.35. The equation $\left(3 x^{2}+1\right)^{2}-\left(P^{2}-4\right) y^{2}=4$ has no integer solutions.

Corollary 3.36. The equation $\left(6 x^{2}+1\right)^{2}-\left(P^{2}-4\right) y^{2}=4$ has positive integer solutions only when $P=3\left(U_{m}(10,-1)-U_{m-1}(10,-1)\right)$ with $m \geq 1$.

Corollary 3.37. The equation $\left(2 x^{2}-1\right)^{2}-\left(P^{2}-4\right) y^{2}=4$ has positive integer solutions only when $P=2 a^{2}-1$ with a integer or $P=V_{m}(6,-1) / 2$ with $m \geq 1$.

Corollary 3.38. The equation $\left(2 x^{2}+1\right)^{2}-\left(P^{2}-4\right) y^{2}=4$ has positive integer solutions only when $P=2 a^{2}+1$, in which case the only solution is $(x, y)=(a, 1)$ with a integer.

\section{References}

[1] R. T. Bumby, The diophantine equation $3 x^{4}-2 y^{2}=1$, Math. Scand. 21 (1967), 144-148.

[2] J. H. E. Cohn, Squares in some recurrent sequences, Pacific J. Math. 41 (1972), 631-646.

[3] J. P. Jones, Representation of solutions of Pell equations using Lucas sequences, Acta Academia Pead. Agr., Sectio Mathematicae 30 (2003), 75-86.

[4] D. Kalman and R. Mena, The Fibonacci numbersexposed, Math. Mag. 76 (2003), no. 3, $167-181$

[5] R. Keskin, Solutions of some quadratics diophantine equations, Comput. Math. Appl. 60 (2010), no. 8, 2225-2230.

[6] R. Keskin and Z. Şiar, Positive integer solutions of some diophantine equations in terms of integer sequences (submitted).

[7] W. L. McDaniel, The g.c.d. in Lucas sequences and Lehmer number sequences, Fibonacci Quart. 29 (1991), no. 1, 24-30. 
[8] — Diophantine representation of Lucas sequences, Fibonacci Quart. 33 (1995), no. $1,58-63$.

[9] R. Melham, Conics which characterize certain Lucas sequences, Fibonacci Quart. 35 (1997), no. 3, 248-251.

[10] J. B. Muskat, Generalized Fibonacci and Lucas sequences and rootfinding methods, Math. Comp. 61 (1993), no. 203, 365-372.

[11] S. Rabinowitz, Algorithmic manipulation of Fibonacci identities, Applications of Fibonacci numbers, Vol. 6 (Pullman, WA, 1994), 389-408, Kluwer Acad. Publ., Dordrecht, 1996.

[12] P. Ribenboim, My Numbers, My Friends, Springer-Verlag New York, Inc., 2000.

[13] P. Ribenboim and W. L. McDaniel, The square terms in Lucas sequences, J. Number Theory 58 (1996), no. 1, 104-123.

[14] Squares in Lucas sequences having an even first parameter, Colloq. Math. 78 (1998), no. 1, 29-34.

[15] - On Lucas sequence terms of the form $k x^{2}$, Number Theory: proceedings of the Turku symposium on Number Theory in memory of Kustaa Inkeri (Turku, 1999), 293-303, de Gruyter, Berlin, 2001.

[16] Z. Şiar and R. Keskin, Some new identities concerning generalized Fibonacci and Lucas numbers, Hacet. J. Math. Stat. 42 (2013), no. 3, 211-222.

[17] _ The square terms in generalized Lucas sequence, Mathematika 60 (2014), 85100.

Mathematics Department

SAKARYA UNIVERSITY

SAKARYA, TURKEY

E-mail address: rkeskin@sakarya.edu.tr 